\title{
Model risk on credit risk
}

\author{
J. Molins and E. Vives* \\ Dept. d'Estructura i Constituents de la Matèria, Universitat de Barcelona, Diagonal 647, Facultat de Física, \\ 08028 Barcelona, Catalonia, Spain
}

\begin{abstract}
This paper develops the Jungle model in a credit portfolio framework. The Jungle model is able to model credit contagion, produce doubly-peaked probability distributions for the total default loss and endogenously generate quasi phase transitions, potentially leading to systemic credit events which happen unexpectedly and without an underlying single cause. We show the Jungle model provides the optimal probability distribution for credit losses, under some reasonable empirical constraints. The Dandelion model, a particular case of the Jungle model, is presented, motivated and exactly solved. The Dandelion model provides an explicit example of doubly-peaked probability distribution for the credit losses. The Diamond model, another instance of the Jungle model, experiences the so called quasi phase transitions; in particular, both the U.S. subprime and the European sovereign crises are shown to be potential examples of quasi phase transitions. We suggest how the Jungle model is able to explain a series of empirical stylized facts in credit portfolios, hard to reconcile by some standard credit portfolio models. We look at model risk in a credit risk framework under the Jungle model, especially in relation to systemic risks posed by doubly-peaked distributions and quasi phase transitions.
\end{abstract}

Keywords: Credit risk, model risk, banking crises, default clustering, contagion, default correlation

\section{Introduction}

Clustering of corporate defaults is relevant for both macroprudential regulators and banks' senior management. With a robust modelling of credit losses, macroprudential regulators may analyse and manage the risk of systemic events in the economy, and banks' senior management may compute the capital needs out of their core credit portfolios.

Historical corporate default rate data, as described in [24] and [10], signal the sensitivity of credit defaults to systemic events in the economy, from the Great Depression and the 2007-2009 Great Recession, to the savings and loans crisis and the burst of the dotcom bubble, as it can be seen from Fig. 1.

Standard credit portfolio models were not able to model the tail risks in credit portfolios when the U.S. subprime and the Spanish Real Estate bubbles bursted. Some of these models introduce default correlations through the dependency of the probabilities of default on macroeconomic factors describing the state of the economy. As a consequence, when the state of the economy is "good", the probabilities of default tend to go down. Conversely, when the state of the economy is "bad", the probabilities of default tend to go up. Aver-

\footnotetext{
*Corresponding author. E-mail: eduard@ecm.ub.edu.
}

aging over the business cycle induces default clustering.

However, these predicted default correlations tend to be low in comparison to empirical evidence, and the corresponding probability distribution of the losses shows "thin tails". It is widely accepted that in addition to the dependence on macroeconomic risk factors, a reasonable credit risk model should include contagion effects, too.

Contagion effects should often give rise to doublypeaked probability distributions for the credit losses, with the first peak being close to the peak of an equivalent binomial distribution (when contagion effects are weak, and the defaults can be considered as roughly independent, which is usually the case when the state of the economy is "good") and a second peak, at higher losses, corresponding to avalanches/domino effects of credit defaults due to contagion.

This paper has the purpose to show a particular class of credit risk model, the Jungle model, ${ }^{1}$ is able to:

\footnotetext{
${ }^{1}$ The name "Jungle" provides intuition for the complex network of dependencies among the constituents of a credit portfolio. Moreover, since the lion is the King of the Jungle, we will see the Dandelion model (from the French "dent-de-lion", or lion's tooth) is the King of the Jungle of contagion models, since the Dandelion may describe the contagion arising from banks, which are the main source of systemic risks.
} 


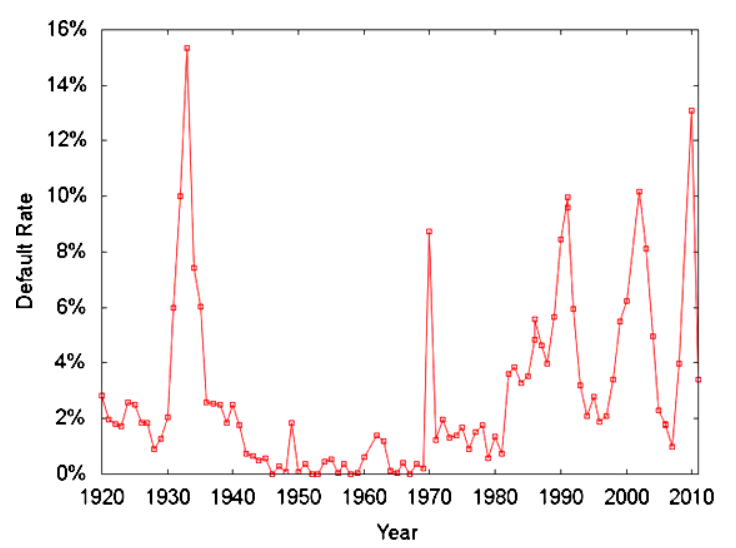

Fig. 1. Historical default rates for Global Speculative-Grade bonds, from [24].

(i) Model contagion among borrowers.

(ii) Endogenously generate doubly-peaked probability distributions for the credit losses. As opposed to the case of single-peaked probability distributions, for which higher credit losses are always less likely than lower losses (at the large loss regime), doubly-peaked probability distributions show the distressing phenomenon that very large losses may be more likely to happen than moderately large losses.

(iii) Show how credit systemic events may occur suddenly and unexpectedly. A credit portfolio may inadvertently cross a "quasi phase transition point", and its collective behaviour change all of a sudden, potentially creating systemic events. We want to emphasize that intuition usually tells us a systemic crisis requires a strong single cause originating it; however, this is not necessarily true. We will show a systemic crisis can be created without a strong underlying, single cause, and we will learn how to recognize those "quasi phase transition points".

Section 3 presents the Jungle model and shows the Jungle model is the optimal probability distribution for modelling losses in a general credit portfolio, under two assumptions:

(i) The Maximum Entropy principle (to be described in Section 3) is the right guiding principle to select the probability distribution of losses in the framework of credit risk modelling.

(ii) All the empirical information of a given credit portfolio can be summarized as probabilities of default and default correlations of its constituents.
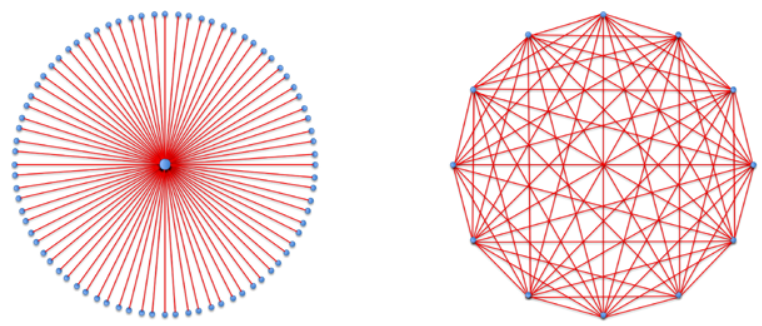

Fig. 2. A Dandelion and a Diamond.

Section 4 tries to motivate the use of the Jungle model. In particular, we show that when there is no empirical information available on default correlations, the Jungle model becomes the binomial distribution (as it should). Section 4 continues with the presentation of a two particular cases of the Jungle model, ${ }^{2}$ the Dandelion model and the Diamond model, both of them are interacting models through contagion, see Fig. 2.

The Dandelion model assumes a central element in the credit portfolio is connected through contagion with the rest of the nodes in the portfolio, and no other pair of nodes is connected. Intuitively, the Dandelion model mimics the relationship between a bank and its many borrowers, or even between a Central Bank with the rest of the economy, see [25].

We show the Dandelion model displays a doublypeaked loss distribution, endogenously generated through contagion. We also find the results of this model can be interpreted as an endogenously generated two-valued mixture model: the two states of the central node can be understood as the two states of the economy, with the probability of default in the "bad" state of the economy being higher than the probability of default in the "good" state of the economy, by an amount given by the variable representing contagion. In a sense, the Dandelion model provides a unifying way to think about both contagion and macroeconomic risk factors.

We argue the Diamond model experiences a quasi phase transition for a not unreasonable set of empirical parameters, showing quantitatively that a small change in the empirical data may result in significant changes for the profile of the probability loss distribution, leading to severe systemic risks, of the kind qualitatively described in [30] and [31].

In Section 5, we provide a series of policy implications arising from our contagion models. In particular, we show both the U.S. subprime and the Euro-

\footnotetext{
${ }^{2}$ An expanded version of the article has been posted at arXiv: 1502.06984
} 
pean peripheral crises can be understood as particular instances of quasi phase transitions. Also, we are able to understand qualitatively other empirical evidence, such as the thick tails in the historical probability distributions of credit losses presented in Section 2, as well as the surprising fact that quite often, the worst quality credit portfolios end up with default rates lower than the corresponding ones with a better rating.

The final section concludes with a summary of the results.

\subsection{Related literature}

Recent literature suggest there are three main sources of credit clustering: macroeconomic risk factors, contagion and frailty.

Macroeconomic risk factors, such as S\&P 500 returns or short term rates, are common to all credits in the portfolio. When the economy grows strongly, the conditional probabilities of default are low. On the contrary, when the economy weakens, the conditional probabilities of default increase. The passage in time of the business cycle induces in a natural way a correlation among credits. Many standard credit portfolio models can be understood as particular instances of a mixed binomial model, see [8].

References [2] and [5] reject the hypothesis that macroeconomic risk factors are able to fully explain the clustering of corporate defaults by themselves, even though [19] argues on the contrary.

Contagion can be understood as direct links among credits, such as the ones in a supply chain, or the bankcreditor relationship. A financial crisis may be a prototypical case of contagion, since banks tend to be highly connected with large parts of the economy, and their financial failure may create a deleveraging, impacting directly on the balance sheet of their borrowers. Contagion was analysed with a dynamical approach in $[6,11,13,21,28,29]$ and in an Ising setting by [23], followed by [16] and [9].

Frailty can be described as the "Enron effect": once the disputable accounting practices were revealed to the public, the probabilities of default of many other companies, in different sectors and regions, readjusted according to the new information. Most likely, no direct links out of contagion between Enron and those companies ever existed, but default correlations arose nonetheless.

References [2,7,19] and [17] include frailty, contagion or both in order to try and explain the clustering of corporate defaults, on top of macroeconomic risk factors. References [2] and [17] conclude both frailty and contagion are necessary to fully explain the clustering of corporate defaults in their datasets, on top of the macroeconomic risk factors.

This paper differentiates from the rest of the literature on contagion networks in the credit arena by arguing its results are independent from the specific details of the "microscopic" credit interactions. In particular, the Maximum Entropy principle argues that given a set of empirical moments of an, in principle, unknown probability distribution, the "best" probability distribution is the Jungle model. The relevant empirical data in the credit arena is known by market participants to be probabilities of default and default correlations. We show that assuming both the probabilities of default and the default correlations correspond to the empirical moments of the unknown probability distribution of credit losses, the Jungle model arises "naturally" and without the need to impose the specific knowledge of the credit interactions among the constituents of the considered credit portfolio.

\subsection{The data}

We use Moody's All rated Annual Issuer-Weighted Corporate Default Rates, from 1920 to 2010, see [24], and [10] value-weighted default rates on bonds issued by U.S. domestic nonfinancial firms from 1866 to 2008.

As often discussed in the literature and among practitioners, default rate data tends to have issues regarding its interpretation as default losses. This is even more the case for such a long term data set as the ones we use. Our approach is pragmatic: [24] and [10] data are no-nonsense, since even though the data definition process is probably not "rigorous" enough (and it cannot be), the data probably is robust enough (the data contains several full business cycles in both cases).

One of the reasons we use a longer data set than is customary in the literature ([2] uses data starting on 1970 ; [5] on 1979; [19] on 1982; [7] on 1979) is that our models do not require the use of macroeconomic or firm-specific data, so we can go backwards as far as we want, while there is still default rate empirical data. On the contrary, for example the S\&P 500 was launched on 1957, so a researcher needs a lot of ingenuity to be able to find the corresponding macroeconomic and firm-specific data corresponding to several decades ago.

From [24], using the default rate for speculative grade bonds, as well as the number of defaults corre- 
sponding to speculative grade bonds, we are able to compute approximately the total amount of speculative grade bonds for each year. For the rest of the paper, when we try to model speculative grade bonds, we will use the average number, close to 800 .

Unfortunately, the corresponding data for single ratings is not provided in the paper. As a consequence, when we deal with Caa-C ratings, we arbitrarily reduce the number for speculative grade bonds by one order of magnitude, 80 .

\section{Credit portfolio modelling}

A credit portfolio consists of $N$ credit instruments. A credit portfolio model is a theoretical construct providing as an output the unconditional probability distribution for the losses of a given credit portfolio (the unknown we will focus our attention on for the rest of the paper).

Moody's KMV [22], CreditMetrics [12], CreditRisk+ [4] and CreditPortfolioView [33] are commercially available credit portfolio models. Additionally, the Gaussian copula [20] became a widespread tool to value credit derivatives. None of these credit portfolio models were able to model tail risks adequately during either the U.S. subprime crisis or the sovereign and banking crisis in peripheral Europe. Especially, some observers believe the Gaussian copula was a "recipe for disaster", see $[15,27]$, when modelling credit tail risks.

The losses of a credit portfolio can be calculated as $L=\sum_{i=1}^{N} L_{i}=\sum_{i=1}^{N} E_{i}\left(1-R R_{i}\right) l_{i}$, where $E_{i}$ denotes the Exposure at Default, i.e. the maximum potential loss out of the credit instrument $i$ (usually, the nominal of the bond or loan), $\left(1-R R_{i}\right)$ denotes the Loss Given Default ( $R R$ stands for Recovery Rate) describing the fraction of the Exposure at Default that is effectively lost when the $i$ th borrower defaults, and $l_{i}$ is an indicator taking values in $\{0,1\}$, and which describes if the $i$ th borrower is defaulted or not.

In general, real world cases, $l_{i}$ variables are stochastic, as well as the recovery rates, and the portfolio is inhomogeneous (in general, $E_{i} \neq E_{j}$ for at last some $i \neq j$ ). The modelling for the related probability distribution of losses is challenging.

We will state our credit portfolio model has been solved when we have found the probability distribution for the losses of that portfolio, $L$. Our target for the rest of the paper will be to motivate, calculate and analyse the probability distribution of $L$.
In this paper and for easiness of presentation, we will make the assumption of analysing homogeneous portfolios (with Exposure at Default set at 1), and we will not model Recovery Rates (which is analogous to assume the Recovery Rates are constant and the same for all borrowers). However, our methodology can equally cope with the general case of inhomogeneous portfolios and state-dependent Recovery Rates, by using standard numerical methods in Statistical Physics. As a consequence and for the rest of the paper, the state space simplifies to a set of discrete variables taking values 0 or $1, \Omega=\left\{\left(l_{1}, l_{2}, \ldots, l_{N}\right) \mid\right.$ $\left.l_{i} \in\{0,1\}, i=1,2, \ldots, N\right\}$. The loss simplifies to $\ell=\sum_{i=1}^{N} l_{i}$.

The probability distribution of a random variable is, in general, difficult to estimate from empirical data. One possible way to derive it is to aggregate the dynamical, "microscopic" processes underlying the random variable. For example, modern physics has been successful at stating microscopic dynamical laws from first principles (quantum mechanics and quantum field theory), and finding the related macroscopic equations (thermodynamics) through an averaging process called Statistical Mechanics.

However, in social sciences this process is fraught with difficulties. In general, the underlying dynamical processes are unknown. The usual methodology then is as follows:

The probability distribution of a random variable is hard to observe per se. But there are observables of the random variable which can be understood as direct calculations from using the probability distribution. For example, the expected value of the random variable is the first moment of the corresponding probability distribution. The variance and the correlation correspond to the second moments of the probability distribution. Skewness and kurtosis, which are widely used empirical observables, are the third and fourth moments of the distribution.

A mathematically well behaved probability distribution can be fully described by its moments. In particular, our underlying random variable, the loss in a credit portfolio, is a bound variable, so we are not concerned about the possibility of moments becoming infinite when the tail of an unbound probability distribution is "fat enough", see [3]. As a consequence, it makes sense to assume that despite the probability distribution being not observable, an analyst may finally recover it through the empirical knowledge of its moments (or in general, through the knowledge of the expected value of a general function; the moments are expected values of polynomials). 
The question is then: given the knowledge of all or some of its moments, is there a way to find the general form of the probability distribution of the underlying random variables?

The Maximum Entropy principle, or Maxent, provides a specific answer to this question. Maxent asserts:

Given a finite state space $\Omega$, the probability distribution in $\Omega$ that maximizes the entropy and satisfies the following $m<\operatorname{card}(\Omega)$ constraints, given $m$ different functions in $\Omega, f_{k}(x)$, and $m$ fixed numbers $F_{k},\left\langle f_{k}(x)\right\rangle:=\sum_{x \in \Omega} P(x) f_{k}(x)=F_{k}, k=$ $1,2, \ldots, m$, as well as a normalization condition $\langle 1\rangle:=\sum_{x \in \Omega} P(x)=1$, is:

$$
P(x)=\frac{1}{Z\left(\lambda_{1}, \lambda_{2}, \ldots, \lambda_{m}\right)} e^{-\sum_{i=1}^{m} \lambda_{i} f_{i}(x)},
$$

where $Z$ is called the partition function, $Z\left(\lambda_{1}, \lambda_{2}, \ldots\right.$, $\left.\lambda_{m}\right)=\sum_{\Omega} \exp \left(-\sum_{i=1}^{m} \lambda_{i} f_{i}(x)\right)$.

The Lagrange multipliers $\lambda_{i}$ are found by inverting the set of $m$ equations $F_{k}=\left\langle f_{k}(x)\right\rangle=$ $-\frac{\partial \log Z\left(\lambda_{1}, \lambda_{2}, \ldots, \lambda_{m}\right)}{\partial \lambda_{k}}, k=1,2, \ldots, m$.

The intuition behind Maxent is $P(x)$ is the "best"3 probability distribution an analyst can come up with, assuming all the empirical evidence about the problem at hand is summarized as expected values of functions (the $F_{k}$ numbers and the $f_{k}(x)$ functions, respectively). The expected values are taken over the (unknown) probability distribution $P(x)$.

It often happens that while the "real" probability distribution of a given system is unknown, some constraints are naturally known. For example, in the trivial case of throwing a dice, we know that whatever the correct probability distribution is, the probabilities for each state (each of the six faces of the dice) must add up to one. In fact, Maxent for the dice gives a uniform probability distribution, with a $p=\frac{1}{6}$ for each of the faces of the dice.

In the same way, if we know, in addition to the fact that all probabilities must add up to one, the expected value of the random variable, Maxent produces the binomial distribution. We will see below that when, in addition to the fact that all probabilities must add up to one, both the expected value of the random variable and its correlations are known, Maxent gives the Jungle model.

Maxent is a general principle which pervades science, see [14]. As a consequence, we feel comfortable

\footnotetext{
${ }^{3}$ We leave the "best" concept undefined
}

enough by stating that Maxent is a reasonable principle to pick the probability distribution of losses for a credit portfolio, consistent with the available empirical data.

Let us apply Maxent to a given credit portfolio:

Our state space is $\Omega=\left\{\left(l_{1}, l_{2}, \ldots, l_{N}\right) \mid l_{i} \in\right.$ $\{0,1\}, i=1,2, \ldots, N\}$, a set of discrete variables, $l_{i}$, taking 0 or 1 values, and representing the default/nondefault state of the $i$ th credit. As a consequence, the potential moments we might derive from the (unknown) probability distribution of losses are:

- The first order moment, $\left\langle l_{i}\right\rangle$. This is the so called probability of default of the $i$ th borrower, $p_{i}$.

- The second order moment, $\left\langle l_{i} l_{j}\right\rangle$, for $i \neq j$. This is directly related to the so called default correlation between the $i$ th and $j$ th borrower, $\rho_{i j}=$ $\frac{q_{i j}-p_{i} p_{j}}{\sqrt{p_{i}\left(1-p_{i}\right)} \sqrt{p_{j}\left(1-p_{j}\right)}}, q_{i j}:=\left\langle l_{i} l_{j}\right\rangle$.

- The second order moment, $\left\langle l_{i} l_{j}\right\rangle=\left\langle l_{i}^{2}\right\rangle$, for $i=j$. However, since $l_{i}$ only takes values in $\{0,1\}$, it is true that $l_{i}^{2}=l_{i}$, so the knowledge of this second moment becomes irrelevant.

- In general, any power of $l_{i}, l_{i}^{k}$, with $k$ being a natural number, becomes $l_{i}$.

- The third order moment, $\left\langle l_{i} l_{j} l_{k}\right\rangle$, for $i \neq j \neq k$, would correspond to the effect on the creditworthiness of the $i$ th borrower, assuming both the $j$ th and $k$ th borrower also default. This effect is conceivable in theory. However, and as far as we know, there is no serious discussion of this phenomenon in the credit literature.

- Any moment of order higher than three is bound to the same discussion as the one for the third order moment above.

There is a general consensus among practitioners that the corresponding available empirical information for a credit portfolio can be summarized as:

- The probability of default of a borrower can generally be estimated, either from CDS for liquid names, or from Internal Ratings models for illiquid bonds or loans. Estimates tend not to be too noisy.

- The default correlation between two borrowers is harder to estimate that the corresponding probabilities of default. There are no financial instruments similar to the CDS to imply the default correlation, or if there are, they tend to be illiquid and over-the-counter (opaque information), and providing noisy estimates. Having said that, and despite the practical difficulties for its estimation, the consensus is the default correlation exists, it 
can at least be measured in some cases, and it is a key variable to understand default clustering.

- Third, and higher, order moments bear no specific names in the credit arena.

As a consequence, we claim that (at least in our mental framework, which consists of disregarding dynamical, "from-first-principle" equations, and only considering probability distributions arising from imposing empirical constraints to Maxent) the empirical available information for credit portfolios can be summarized in the probabilities of default and default correlations of its constituents.

Maxent selects the Jungle model as its preferred probability distribution for credit losses, consistent with the available empirical data, as seen in the next section.

\section{The Jungle model and credit risk}

We consider a credit portfolio of $N$ credit instruments, with a space state $\Omega=\left\{\left(l_{1}, l_{2}, \ldots, l_{N}\right) \mid l_{i} \in\right.$ $\{0,1\}, i=1,2, \ldots, N\}$.

We consider the set "labeling" the $N$ nodes, $\Theta=$ $\{1,2, \ldots, N\}$ and the set "labelling" the $\frac{N(N-1)}{2}$ pairs of nodes, $\Phi=\{(i, j) \mid i=1,2, \ldots, N \& j>i\}$ (the pair $i j$ and the pair $j i$ are considered to be the same), and two subsets of those, $\theta \in \Theta$ and $\phi \in \Phi$.

In consistency with the previous section, we assume the full available empirical information of the corresponding credit portfolio can be summarized as the probabilities of default and the default correlations of its constituents.

We will always consider $\theta=\Theta$, or in other words, we assume it is possible to give estimates of the probabilities of default for all the constituents in the portfolio, but $\phi$ will usually be a proper subset of $\Phi$, meaning some of, but not all, the default probabilities can be estimated. The general case will be one in which $1 \ll \operatorname{card}(\phi) \ll \frac{N(N-1)}{2}$.

Using the framework of Maxent, we claim that given the following empirical data, consisting of default probabilities and default correlations:

- $p_{i}, \forall i \in \theta$, with $p_{i} \in[0,1]$.

- $\rho_{i j}, \forall(i, j) \in \phi$, with $\rho_{i j} \in[-1,1]$; we define $q_{i j}$ such that the relationship $\frac{q_{i j}-p_{i} p_{j}}{\sqrt{p_{i}\left(1-p_{i}\right)} \sqrt{p_{j}\left(1-p_{j}\right)}}=$ $\rho_{i j}, \forall(i, j) \in \phi$ holds.
Leading to the following empirical constraints:

- $p_{i}=\left\langle l_{i}\right\rangle, \forall i \in \theta$.

- $q_{i j}=\left\langle l_{i} l_{j}\right\rangle, \forall(i, j) \in \phi$.

Maxent picks the Jungle model among all the probability distributions consistent with those constraints: ${ }^{4}$

$$
P\left(l_{1}, l_{2}, \ldots, l_{N}\right)=\frac{1}{Z} e^{\sum_{i \in \theta} \alpha_{i} l_{i}+\sum_{(i, j) \in \phi} \beta_{i j} l_{i} l_{j}},
$$

where $Z=\sum_{\Omega} \exp \left(\sum_{i \in \theta} \alpha_{i} l_{i}+\sum_{(i, j) \in \phi} \beta_{i j} l_{i} l_{j}\right)$.

The unknown parameters $\alpha_{i}$ and $\beta_{i j}$ have to be found by forcing the probability distribution gives the right estimates for the empirical information at our disposal, i.e. the constraints $p_{i}=\left\langle l_{i}\right\rangle=\frac{\partial \log Z}{\partial \alpha_{i}}$ and $q_{i j}=\left\langle l_{i} l_{j}\right\rangle=\frac{\partial \log Z}{\partial \beta_{i j}} \forall i \in \theta$ and $\forall(i, j) \in \phi$.

\section{The Jungle model, hands on}

After showing the Maxent principle picks the Jungle model as the probability distribution of choice to analyse credit risk (assuming the full empirical information of the credit portfolio can be summarized as the probabilities of default and the default correlations of its constituents), we try and motivate the Jungle model, by studying some particular instances of the general model.

To accomplish that goal, this section presents a few particular cases of the general Jungle model: the binomial model, the Dandelion model and the Diamond model.

In this section, we will show the Jungle model is able to introduce credit contagion in a way the standard credit portfolio models cannot. In particular, the Jungle model will be shown to model credit correlations under "normal economic conditions" (in a similar way to a Gaussian copula, at least at a non-quantitative level of discourse), but also to endogenously generate quasiphase transitions, which can be understood as modelling systemic credit crises, arising "out of nowhere", a phenomenon that by definition, a Gaussian copula cannot cope with (in other words, a model under a Gaussian copula never suffers from systemic crises).

\footnotetext{
${ }^{4}$ In the physics literature, the Jungle model is called the Ising model with external field, with both space dependent external fields and space dependent local interactions.
} 


\subsection{The binomial model}

For a credit portfolio whose probabilities of default are known and equal to each other, $\left\{p_{i}:=p \mid i=\right.$ $1,2, \ldots, N\}$ but whose default correlations $\left\{\rho_{i j} \mid i=\right.$ $1,2, \ldots, N \& j \neq i\}$ are unknown, the probability distribution chosen by the Maximum Entropy principle is $P\left(l_{1}, l_{2}, \ldots, l_{N}\right)=\frac{1}{Z} \exp \left(\alpha \sum_{i=1}^{N} l_{i}\right)$.

Due to homogeneity, the distribution above becomes the binomial distribution:

$$
P\left(\sum_{i=1}^{N} l_{i}=\ell\right)=\left(\begin{array}{l}
N \\
\ell
\end{array}\right) p^{\ell}(1-p)^{N-\ell}
$$

with the identification $p=\frac{1}{1+e^{-\alpha}}$. In other words, for the uncorrelated portfolio, the parameter $\alpha$ can be interpreted as (a simple function of) the probability of default.

Since the binomial distribution corresponds to independent defaults, it makes intuitive sense the Maximum Entropy principle selects it when there is no information whatsoever on empirical correlations.

\subsection{The Dandelion model}

The Dandelion model corresponds to a Jungle model with $N+1$ borrowers, such that the first one, defined as $i=0$ and considered to be at the centre of the Dandelion, is "connected" to all remaining borrowers, at the external surface of the Dandelion, such that $\beta_{0 i}=: \beta \neq 0$ for $i=1,2, \ldots, N$. Any other borrowers remain unconnected, $\beta_{i j}=0$ for $i=1,2, \ldots, N$ $\& j>i$. For simplicity, we assume $\alpha_{i}=: \alpha$ for $i=1,2, \ldots, N$.

The probability distribution for the Dandelion model is $P\left(l_{1}, l_{2}, \ldots, l_{N}\right)=\frac{1}{Z} \exp \left(\alpha_{0} l_{0}+\alpha \sum_{i=1}^{N} l_{i}+\right.$ $\left.\beta \sum_{i=1}^{N} l_{0} l_{i}\right)$.

The Dandelion model, despite being interacting, can be fully solved, with the probability distribution for its losses given by:

$$
P\left(\ell=\sum_{i=1}^{N} l_{i}\right)=\frac{1}{Z}\left(\begin{array}{l}
N \\
\ell
\end{array}\right)\left(e^{\alpha \ell}+e^{\alpha_{0}+\ell(\alpha+\beta)}\right),
$$

where $\mathrm{Z}$ is given by $Z=\left(1+e^{\alpha}\right)^{N}+e^{\alpha_{0}}\left(1+e^{\alpha+\beta}\right)^{N}$, and $\alpha_{0}=(N-1) \log \left(\frac{1-p_{0}}{p_{0}}\right)+N \log \left(\frac{p_{0}-q}{1-p_{0}-p+q}\right)$,

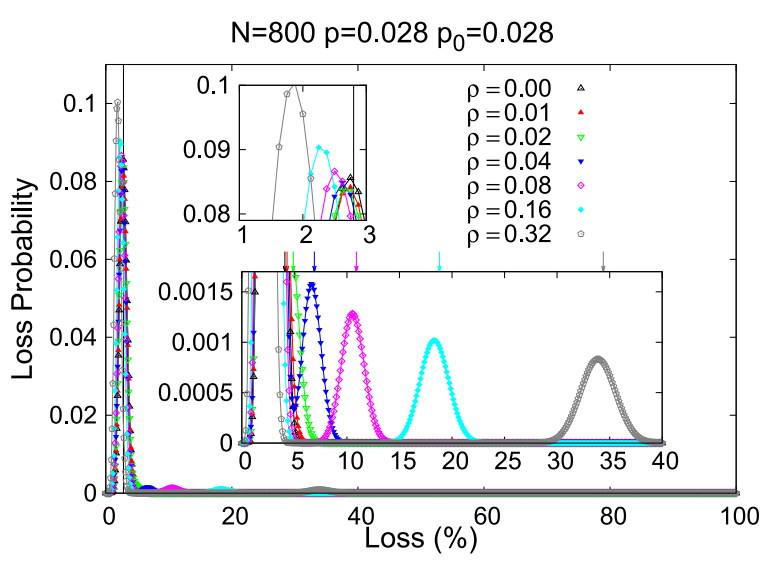

Fig. 3. Probability distributions for the losses of the Dandelion model, corresponding to different default correlations.

$\alpha=\log \left(\frac{p-q}{1-p_{0}-p+q}\right)$ and $\beta=\log \left(\frac{q}{p_{0}-q} \frac{1-p_{0}-p+q}{p-q}\right)$, where $q$ can be derived from the definition of default correlation, $\rho=\frac{q-p p_{0}}{\sqrt{p(1-p)} \sqrt{p_{0}\left(1-p_{0}\right)}}$.

To provide intuition for the Dandelion model, we have calculated its probability distribution for a set of reasonable parameters, $N=800$ and $p=p_{0}=2.8 \%$, which correspond to the historical default rate average for global speculative-grade bonds, as per [24], and for a given range of possible default correlations. The result can be found in Fig. 3 .

The probability distributions in the picture show a "double peak" pattern: on one hand, a first peak, centred at low losses and not unlike the corresponding peak for a binomial distribution. On the other hand, a smaller but not insignificant second peak, corresponding to a high level of losses, and consistent with avalanches/domino effects due to contagion.

The higher the default correlation, the higher the extreme losses (the second peak moves further to the right on the chart). Also, the higher the default correlation, the lower losses on the first peak. Contagion works both ways: defaults lead to more defaults (with respect to the binomial case), non-defaults lead to more non-defaults (with respect to the binomial case). These two effects can be seen more specifically from the two insets in the chart.

The Dandelion model can be understood as a bridge between macroeconomic risk factors and contagion. Specifically, in the derivation of the Dandelion model, the following equation arises:

$$
p=\left(1-p_{0}\right) p(\alpha)+p_{0} p(\alpha+\beta)
$$


where $p(\alpha)=\frac{1}{1+e^{-\alpha}}$ corresponds to the relationship between $p$ and $\alpha$ described for the binomial (noninteracting) case.

As a consequence, the central node in the Dandelion could be interpreted as endogenously generating a "macroeconomic state of the economy", whereby for a fraction of time given by $1-p_{0}$ the economy remains in a "good" state of the economy, with a probability of default for its constituents given by $p(\alpha)=\frac{1}{1+e^{-\alpha}}$, and for a fraction of time given by $p_{0}$ the economy remains in a "bad" state of the economy, with a probability of default for its constituents given by $p(\alpha+\beta)=$ $\frac{1}{1+e^{-(\alpha+\beta)}}$, where $p(\alpha+\beta)>p(\alpha)$, and the difference is accounted by the "contagion factor" $\beta$.

In other words, the Dandelion model endogenously generates a kind of mixture of binomials, able to generate a doubly peaked distribution and clustering of defaults.

\subsection{The Diamond model}

The Diamond model is defined by $Z=$ $\sum_{l_{1}, l_{2}, \ldots, l_{N}} \exp \left(\alpha \sum_{i=1}^{N} l_{i}+\beta \sum_{i>j} l_{i} l_{j}\right)$, and it was first analysed in [23]; see also [1] and [26].

The Diamond model describes a set of credits, all interacting among each other. For example, if $N=4$, node 1 could be a bank, node 2 a cement producer, node 3 a real estate developer and node 4 , a car dealer. The cement producer, the real estate developer and the car dealer get financing from the bank, so there are correlations of defaults between the pairs 12,13 and 14. Also, the cement producer is a supplier to the real estate developer, so the pair 23 is also correlated. Finally, workers at firms 2 and 3 purchase cars from the car dealer, so a default of 2 or 3 would impact on 4 business, creating also default correlations between 24 and 34 .

The partition function for the Diamond model is given by:

$$
Z=\sum_{\ell=0}^{N}\left(\begin{array}{l}
N \\
\ell
\end{array}\right) e^{\left(\alpha-\frac{\beta}{2}\right) \ell+\frac{\beta}{2} \ell^{2}} .
$$

And the corresponding probability distribution for the losses will be:

$$
P\left(\ell=\sum_{i=1}^{N} l_{i}\right)=\left(\begin{array}{l}
N \\
\ell
\end{array}\right) \frac{e^{\left(\alpha-\frac{\beta}{2}\right) \ell+\frac{\beta}{2} \ell^{2}}}{Z} .
$$

We can relate the empirical data, $p$ and $\rho$ to the model parameters $\alpha$ and $\beta$, from the following two equations which can be inverted numerically:

$$
\begin{aligned}
p= & \frac{1}{Z N} \sum_{\ell=0}^{N}\left(\begin{array}{l}
N \\
\ell
\end{array}\right) \ell e^{\left(\alpha-\frac{\beta}{2}\right) \ell+\frac{\beta}{2} \ell^{2}}, \\
q= & \frac{2}{Z N(N-1)} \\
& \times \sum_{\ell=0}^{N}\left(\begin{array}{l}
N \\
\ell
\end{array}\right) \frac{1}{2} \ell(\ell-1) e^{\left(\alpha-\frac{\beta}{2}\right) \ell+\frac{\beta}{2} \ell^{2}} .
\end{aligned}
$$

The Diamond model clearly exemplifies one of the most interesting phenomena of the Jungle model: quasi phase transitions.

Let us see how the probability distribution of losses for the Diamond model changes, when we smoothly change default correlations, for the probability of default fixed at a given level (with parameters $N=20$ and $p=40 \%$, for easiness of visual inspection; below, we will provide another example, with $N=50$ and $p=2.8 \%$ ), as shown in Fig. 4:

We can see there is a sudden change of collective behaviour for the probability distribution of losses when we smoothly change $\rho$ from $10 \%$ to $20 \%$ to $30 \%$, at some point between these default correlations:

For default correlations at around $10 \%$ or below, the Diamond model presents a standard behaviour with losses spread with a given width around the expected value, $40 \%$. However, when the default correlation increases only slightly (to $25 \%$, say), a different behaviour for the probability distribution of losses starts to emerge: the probability distribution for the losses becomes bimodal, as it can be seen from Fig. 4. And the more the default correlation increases, the larger the potential losses out of the second peak on the right.

Another numerical example, this time with $N=50$ and $p=2.8 \%$, the average default rate for speculativegrade bonds in the [24] sample, shows how a quasi phase transition changes dramatically the risk profile of the loss probability distribution, given small changes of the empirical values determining the portfolio (probabilities of default and, especially, default correlations):

From Fig. 5, we can see a sudden jump for the Value at Risk at the $99.9 \%$ confidence level, given a small increase in the default correlation. ${ }^{5}$

\footnotetext{
${ }^{5}$ This is the explanation for the use of the "phase transition" concept, borrowed from Statistical Mechanics. Phase transitions suffer
} 

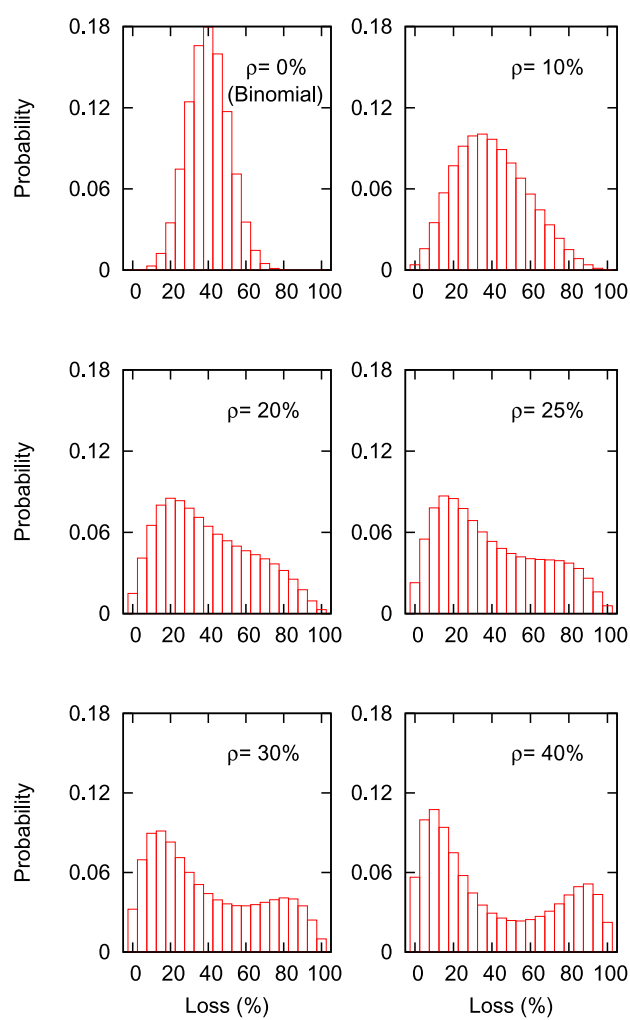

Fig. 4. Loss probability distributions for default correlations below, around and above the quasi phase transition point.

The above phenomenon can also be analysed by looking at how the empirical variables, both the probability of default and the default correlation, change when the model parameters, $\alpha$ and $\beta$, change, as shown in Fig. 6.

Despite the fact that for a finite $N$, there can be no (exactly discontinuous) phase transition, from the figures above we can see a sharp, almost discontinuous behaviour throughout a clearly visible diagonal line in the space of model parameters. By analogy, we give the name "quasi phase transition" to that phenomenon. The existence of such a line, ending in the so called "critical point", 6 is well known in the Condensed Matter physics literature.

a sudden jump in a given variable, induced by a small change in another, underlying variable. However, a quasi phase transition is not a phase transition, as properly defined in Statistical Mechanics. For example, phase transitions for the Ising model, the equivalent of the Jungle model in Physics, cannot happen for finite $N$, and throughout the paper we assume $N$ is always finite.

${ }^{6}$ It is known in the Physics literature the critical point lies at $\alpha=$ $-2, \beta=\frac{4}{N}$ in the large $N$ limit, corresponding approximately to a probability of default of $44 \%$ and a default correlation of $11 \%$ for $N=80$.
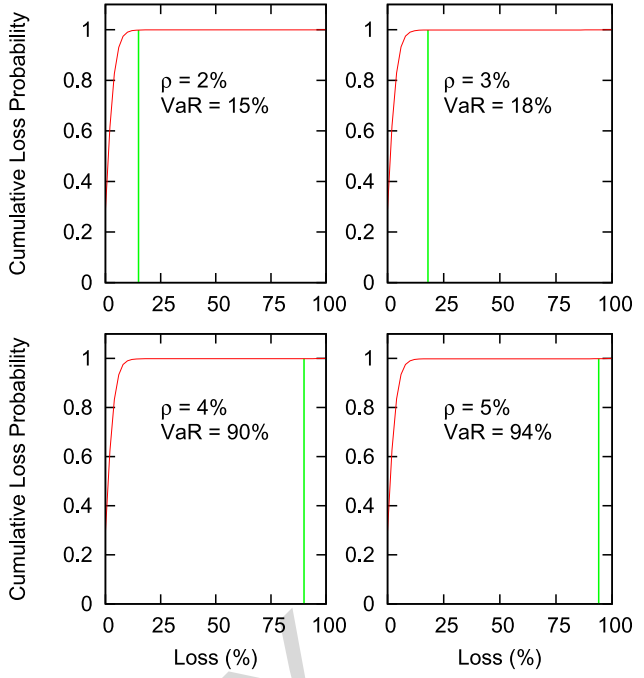

Fig. 5. Cumulative probability loss distributions for default correlations below, around and above the quasi phase transition point.

Since there is a known relationship between the empirical parameters, the probability of default and the default correlation, and the model parameters, $\alpha$ and $\beta$, an analyst can use either the empirical parameters or the model parameters to describe the behaviour of the model. The model parameters tend to be more useful to analyse the behaviour of such systems, because as observed from Fig. 6, a small change in the empirical parameters always results in a small change for the model parameters, but the opposite is clearly not true (at the line of quasi phase transitions):

Also, any credit portfolio is driven by underlying, fundamental economic factors (both macroeconomic and microeconomic). As a consequence, we can understand the evolution in time of a credit portfolio described by such a model as the smooth change of the model parameters when the underlying, fundamental economic factors change. Usually, the empirical parameters will be such that the system will move around the bottom left corner of Fig. 6 (relatively low probabilities of default and default correlations).

However, when the economic conditions are such that the system is close to (but still below) the line of quasi phase transitions, a small change in the underlying, fundamental economic factors may lead to a small change in the model parameters, such that the system may inadvertently cross the line of quasi phase transitions, resulting in an abrupt, almost discontinuous, change in the empirical parameters.

As a consequence, the Diamond model shows antiintuitively that the collective behaviour of the portfolio 

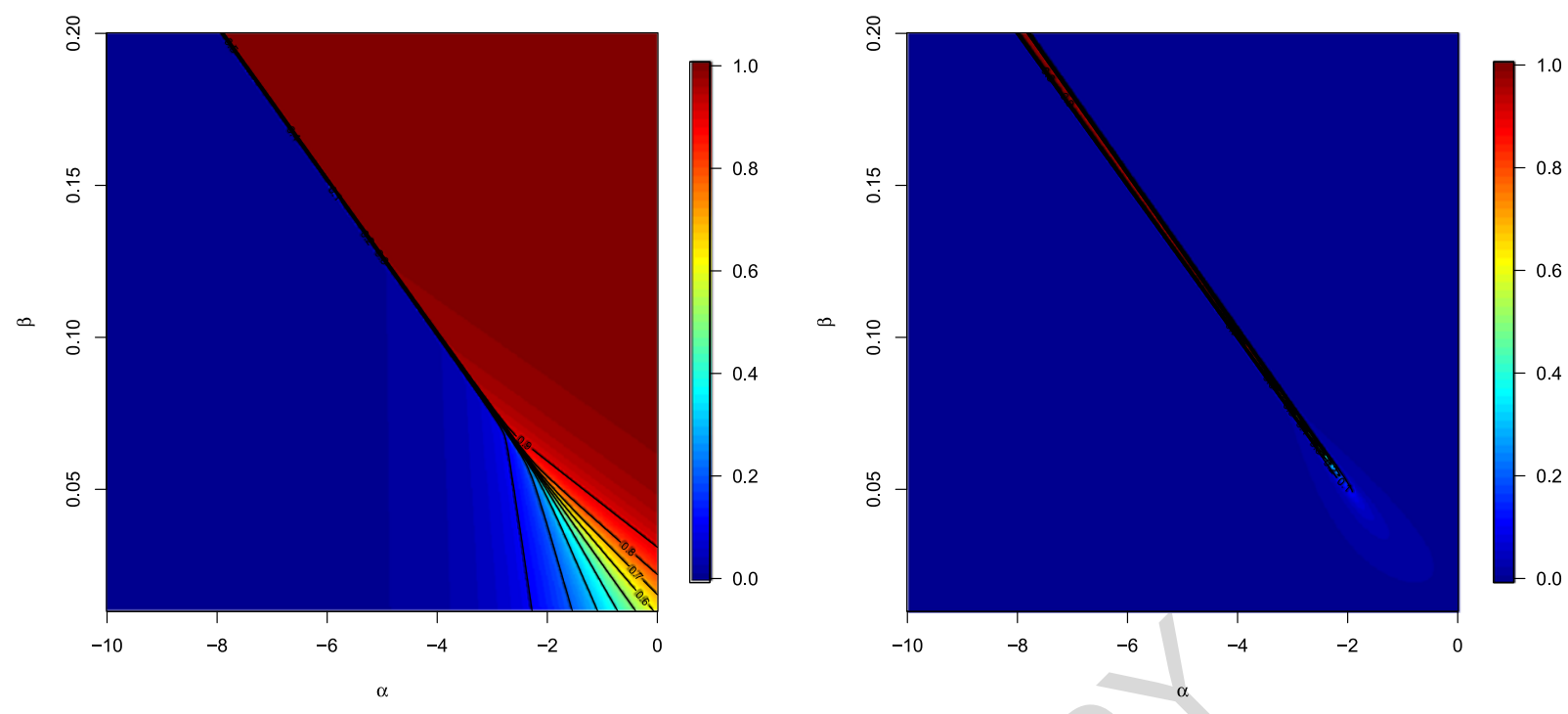

Fig. 6. Probability of default and default correlation for a given set of model (normalized to values between 0 and 1 ) parameters, $\alpha$ and $\beta$, and $N=80$.

may significantly change due to small changes of the empirical values determining it.

This phenomena is not unlike the phase transition of water into steam: if we increase one degree Celsius the temperature of water at 98 degrees Celsius, the resulting water at 99 degrees Celsius continues "being water" (small details will change, for example a thermometer inside the water will show a small increase in its readings, but water will remain "being water"). However, when the temperature increases a further degree Celsius, there is a sudden change in the collective behaviour of water, becoming a coexistence of steam bubbles and liquid.

So, a small change of the underlying parameters leads to a significant change of the behaviour for the whole system. This is surprising, since if we could solve all the dynamical equations of motion for the say $10^{23}$ particles in a litre of water, it seems unlikely that with that knowledge we could have forecasted such a dramatic change of behaviour. It is the averaging out of "irrelevant" degrees of freedom, undertaken by statistical mechanics, which allows to keep only the (small set of) parameters which really matter at the level of one litre of water.

Analogously, the Diamond model shows a quasi phase transition from a phase dominated by a "binomial-like" behaviour, whereby losses spread over a given width, centred around the expected loss, towards a coexistence region, dominated by avalanches due to credit contagion, and determined by a doubly peaked distribution. The transition from one phase to the coexistence region is caused by a smooth change of the empirical parameters defining the portfolio (probabilities of default and default correlations). However, the variation in the global shape of the probability distribution changes significantly the risk profile of the portfolio, potentially inducing systemic risks.

\section{Policy implications of contagion}

In Section 4, we have described how the Jungle model depends on the probabilities of default and default correlations of its constituents, plus the topology of the "contagion network".

We have seen that for several topologies, with nottoo-unreasonable values for the probabilities of default and default correlations, the probability distributions of the credit losses become doubly peaked, out of credit avalanches triggered by contagion.

In particular, we have analysed how increasing the default correlation for the Dandelion model, leads to the second peak moving to more extreme losses (more extreme domino effects), as well as the first peak moving towards zero losses. The inability of some credit portfolio models to accommodate these stylized facts, even for some models used in practice for regulatory purposes such as [32], has been highlighted by [18].

In the following sections, we will describe the policy implications suggested by the effects above. 


\subsection{The U.S. subprime and the European sovereign crises as quasi-phase transitions}

Both the U.S. subprime and the European sovereign crises caused sharp spikes in probabilities of default "across the board". However, such increases were mostly concentrated in specific sectors of the economy (e.g., financial corporates, such as Lehman and AIG, during the U.S. subprime crisis).

For the following argument, it will be important to highlight that not only the probabilities of default jumped during the financial crises, but also the (average pairwise) default correlations, as it can be seen from Fig. 7.

Before the crisis, market expectations implied low probabilities of default and default correlations for the overall economy, in particular the same held true for the financial sector. Also, risk aversion was probably "low", and as a consequence, the risk premium associated to the market values of both empirical parameters was also "low".

However, when the crisis erupted, both probabilities of default and default correlations spiked up for the financial sector as a whole. Even more, if it were possible to define the sub-sector of the most speculative parts of the financial sector (those companies exposed the most to subprime assets), it seems reasonable to assume the probability of default of such sub-sector reached exceedingly high levels, of the order of magnitude of $50 \%$.

From the analysis of the Diamond model, we have seen quasi phase transitions arise naturally. And the Diamond model, with equally pairwise default correlations, is the corresponding Jungle model associated to a homogeneous portfolio whereby all nodes are connected to each other. And the sub-sector of

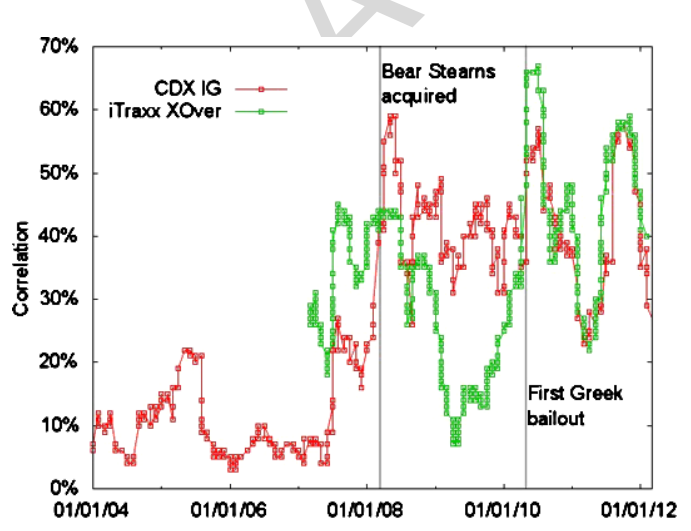

Fig. 7. Historical default correlation for both iTraxx XOver and CDX IG. the most speculative parts of the financial sector constitutes a credit portfolio which probably can be correctly approximated as homogeneous, with each node connected to any other node in the network. In fact, the standard methodology to imply default correlations from traded credit indices, such as CDX IG or iTraxx XOver, assumes all their individual components have the same default correlation with every other name in the index.

Then, for a Diamond model, and under "normal" (non-crisis) conditions, the fundamental situation of the economy is such that both the probabilities of default and the default correlations are "low", resulting in an economy located around the bottom-left corner of Fig. 6. However, in a crisis, and for that sub-sector of the economy exposed the most to the key fundamentals of the crisis, the model parameters $\alpha$ and $\beta$ may get closer to the "line of quasi phase transitions", from below. As we have observed before, the "critical point" of such line corresponds to a probability of default of $44 \%$ and a default correlation of $11 \%$ for a reasonable set of parameters.

As a consequence, if for such a sub-sector, the impact of the deterioration in the fundamental situation of the economy implies that the corresponding probabilities of default and default correlations are close (from below) to $44 \%$ and $11 \%$, resp., just a little bit of further economic deterioration may result in the model parameters, $\alpha$ and $\beta$, crossing up the "line of quasi phase transitions" (from the bottom left corner, to the top right corner). Such a small change may be immaterial in the model parameters space, but in the empirical parameters space, the impact is huge: both the probability of default and the default correlation spike up, from low levels (close to 0\%) to high levels (close to $100 \%)$.

The policy implication of the discussion above is then as follows: monitor closely the model parameters, $\alpha$ and $\beta$, of the sectors of the economy most exposed to potential future systemic crises (the financial sector being always one of such sectors), and raise a red flag once and if the model parameters are getting closer to the "line of quasi phase transitions", or at least, a reasonable stress test suggests the model parameters might cross such line in case of a sudden macroeconomic/microeconomic shock.

In particular, let us highlight that such a policy would have resulted in a "red flag" for both the U.S. subprime and the European sovereign crises at the beginning of such crises, and even possibly a bit before their sudden eruption, as it can be seen intuitively from the default correlation figures above. 


\subsection{Understanding the historical probability distributions of credit losses}

The historical default rates provided by [24] (also data from [10] would allow us to reach similar conclusions) yield the histograms in Fig. 8.

From visual inspection for the three figures, there are too few data points to robustly ascertain if the prob-
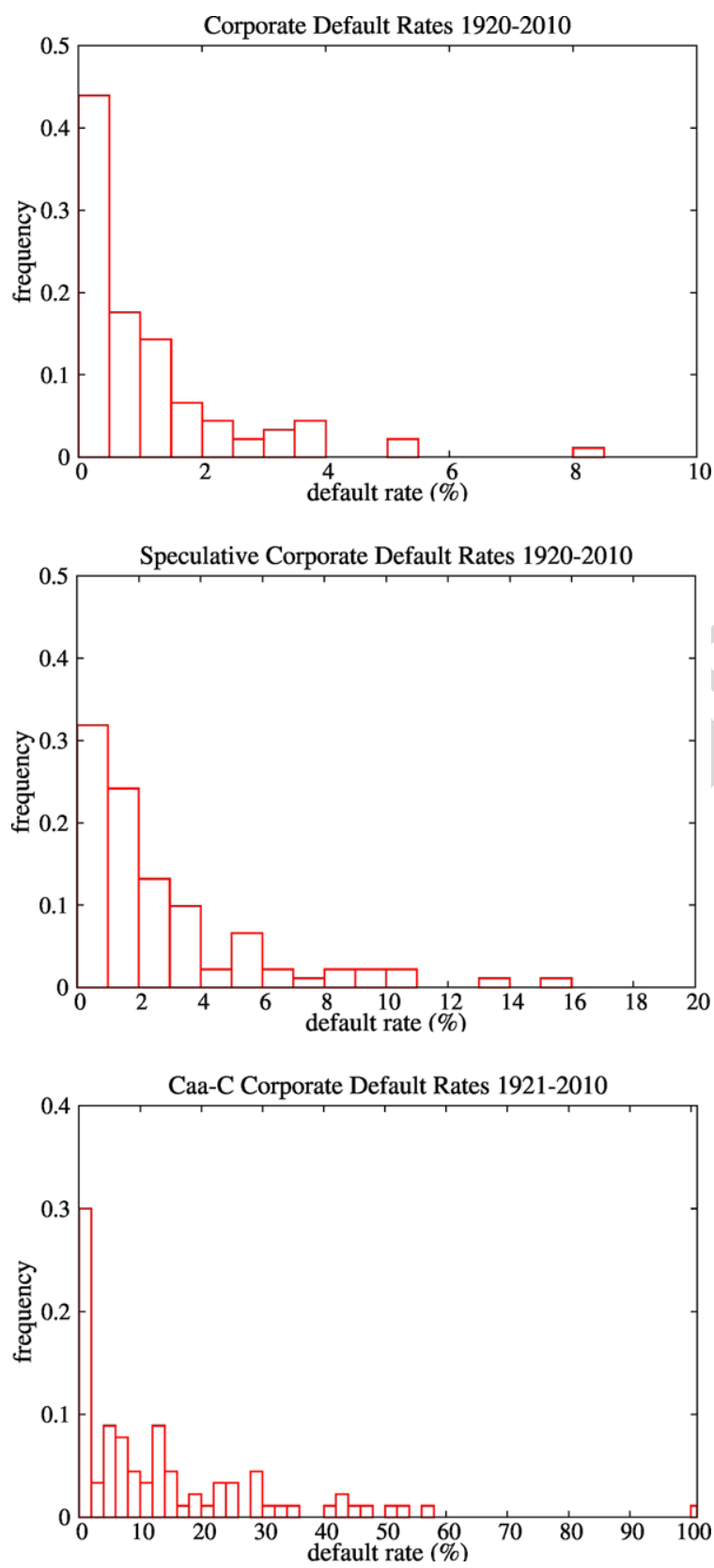

Fig. 8. Histograms for data in [24]. ability distributions for the default rates have one peak or more. Intuitively, it seems the tail is a fat one, with credit loss realizations up to $100 \%$, in the case of Caa$\mathrm{C}$ rating.

The question is then if this evidence contradicts our claim that the fact that Maxent picks the Jungle model as its probability distribution of losses of choice suggests the Jungle model is a reasonable credit risk model to be considered in practice, since the Jungle model is often (for several topologies) doubly peaked, as for example the Dandelion model.

The answer is that it does not. The historical distribution of losses presented above can be understood as follows:

Let us assume, without loss of generality, that the empirical probabilities of default and default correlations only change once per year, on Jan 1st. Let us assume the corresponding topology gives rise to a Jungle model generating doubly-peaked probability distributions. Then, the losses that year will be a realization of that particular Jungle model. Probably, the realization will fall under the first peak. But the more years we repeat the same procedure (with their corresponding probabilities of default, default correlations and topology), the more likely is a realization occurs on the second peak (contagion effects, generating an avalanche/ domino effect of credit defaults).

From the Dandelion model, we have found out the position of the second peak is largely determined by the default correlation (the probabilities of default also matter).

As time passes by, we will have a series of realizations of the second peak. But importantly, the empirical data for each realization (probabilities of default, default correlations and topology) will most likely be different for each year, probably generating a double peak at different location on the axis of losses for each realization of the second peak.

As a consequence, the historical probability distribution of losses will probably have only a first peak, consistent with the fact that in the majority of realizations, losses are basically contagion-less, so that first peak will be roughly similar to the one of a binomial model, but wider due to the mixing with different macroeconomic conditions over several business cycles, and a fat tail generated by realizations of the doubly-peaked probability distributions arising from the Jungle model.

This way of thinking allows us to understand how is it possible the tail of the empirical probability distributions is so "thick": the tail is generated through individual realizations of double peaks. This way of think- 
ing relaxes the need to include extreme probability distributions which are able to cope by themselves with the difficult task to model both extreme default events, and default events in a "good" economy state.

Even more, the Jungle model allows us to understand a stylized fact of the probability distribution of losses for highly risky portfolios, exemplified by Moody's Caa-C rating data: despite the fact Caa-C rating bonds are highly risky (and there is even one year where $100 \%$ of bonds in the sample defaulted), it also happens very often that $\mathrm{Caa}-\mathrm{C}$ rating bonds enjoy a default rate close to $0 \%$ (on the sample, there are several years with $0 \%$ default rate). In fact, this phenomenon of $0 \%$ default rate happens more often for Caa-C than for bonds with a much better rating, which seems intuitively odd, see [18].

However, the Dandelion model is able to explain this stylized fact: for Caa-C rating bonds, it seems likely that the individual bonds are described not only by high probabilities of default, but also by high default correlations among themselves (or with a central node, in a similar way to the Dandelion model; possibly banks or other financial suppliers specialized on risky lending).

From the charts in the Dandelion model section, we can see that in this region of parameters, the higher the default correlation, the larger the losses for the double peak. But in addition to this effect, also the higher the default correlation, the lower the losses for the first peak. This is consistent with a contagion effect: contagion not only works on "bad" situations (a default in a node induces a default in another node nearby), but also on "good" situations (a non-default in a node induces a non-default in another node nearby).

As a consequence, this framework of thinking leads us to suggest that the most relevant variable to ascertain default clustering is not the probability of default (as standard rating classifications appear implicitly to suggest) but default correlations

\section{Conclusions}

This paper presents and develops the Jungle model in a credit portfolio framework. The Jungle model generates a probability distribution for the losses of a credit portfolio with the following stylized facts:

(i) The Jungle model is able to model contagion among borrowers. (ii) The Jungle model endogenously generates doubly-peaked probability distributions for the credit losses, with the second peak consistent with avalanches/domino effects out of contagion.

(iii) The Jungle model endogenously generates quasi phase transitions, meaning small changes in the portfolio may result in sudden and unexpected systemic risks. The Jungle model helps us to ascertain the location and nature of those quasi phase transition points.

We study a series of particular cases of the Jungle model, in particular the Dandelion model and the Diamond model.

The Dandelion model exemplifies the emergence of doubly-peaked probability distributions. The Diamond model quantifies how and when a quasi phase transition may occur for the Jungle model.

Model risk arises from the genuine model uncertainty: potentially, there will be many different Jungle models consistent with the set of available empirical data for our portfolio. As a consequence, by considering the potential systemic risks of this ensemble of Jungle theories allows us to address model risk. In particular, we have shown that for not too unreasonable data, some Jungle models endogenously generate a quasi phase transition, i.e. given small changes in the underlying empirical parameters may induce sudden changes in the collective behaviour of the system, potentially and inadvertently generating systemic events. Quasi phase transitions and doubly-peaked probability distributions represent a challenge for model risk.

The analysis of the Jungle model in general, and of the Dandelion and Diamond models in particular, leads to some policy implications of contagion. We are able to understand qualitatively some empirical evidence, such as the embedding of the U.S. subprime and the European peripheral crises into the general framework of quasi phase transitions, the thick tails in the historical probability distributions, as well as the surprising fact that quite often, the worst quality credit portfolios end up with default rates lower than the corresponding ones with a better rating.

We believe the study of the Jungle model in the credit arena, especially for regulatory purposes, deserves further attention.

\section{References}

[1] T. Antal, M. Droz and Z. Rácz, Probability distribution of magnetization in the one-dimensional Ising model: Effects of boundary conditions, J. Phys. A 37 (2004), 1465-1478. 
[2] S. Azizpour, K. Giesecke and G. Schwenkler, Exploring the sources of default clustering, available at: http://web. stanford.edu/dept/MSandE/cgi-bin/people/faculty/giesecke/ pdfs/exploring.pdf.

[3] J.P. Bouchaud and M. Potters, Theory of Financial Risk and Derivative Pricing, Cambridge University Press, 2003.

[4] CreditSuisse, CreditRisk+: A credit risk management framework, Credit Suisse First Boston, 1997, 71 p.

[5] S. Das, D. Duffie, N. Kapadia and L. Saita, Common failings: How corporate defaults are correlated, J. Finance 62 (2007), 93-117.

[6] M. Davis and V. Lo, Infectious defaults, Quantitative Finance 1(4) (2001), 382-387.

[7] D. Duffie, A. Eckner, G. Horel and L. Saita, Frailty correlated default, J. Finance 64 (2009), 2089-2123. doi:10.1111/ j.1540-6261.2009.01495.x.

[8] P. Embrechts, R. Frey and A. McNeil, Credit risk models: An overview, available at: http://www.math.ethz.ch/ embrecht/ ftp/K.pdf.

[9] I.O. Filiz, X. Guo, J. Morton and B. Sturmfels, Graphical models for correlated defaults, Mathematical Finance 22 (2012), 621-644. doi:10.1111/j.1467-9965.2011.00499.x.

[10] K. Giesecke, I. Strebulaev and S. Schaefer, Corporate bond default risk: A 150-year perspective, Journal of Financial Economics 102 (2011), 233-250.

[11] K. Giesecke and S. Weber, Cyclical correlations, credit contagion, and portfolio losses, Journal of Banking and Finance $\mathbf{2 8}$ (2004), 3009-3036.

[12] G. Gupton, C. Finger and M. Bhatia, CreditMetrics - Technical Document, J.P. Morgan, New York, USA, 1997, 212 p.

[13] R.A. Jarrow and F. Yu, Counterparty risk and the pricing of defaultable securities, J. Finance 56 (2001), 1765-1799.

[14] E.T. Jaynes, Probability theory: The logic of science, Law, Probability and Risk 3(3-4) (2004), 243-246. doi:10.1093/ lawprj/3.3-4.243.

[15] S. Jones, The formula that felled Wall St, Financial Times.

[16] K. Kitsukawa, S. Mori and M. Hisakado, Evaluation of tranche in securitization and long-range Ising model, Physica A: Statistical Mechanics and Its Applications 1 (2006), 191-206.

[17] S.J. Koopman, A. Lucas and B. Schwaab, Modeling frailty correlated defaults using many macroeconomic covariates, Journal of Econometrics 162(2) (2011), 312-325.
[18] P.H. Kupiec, How well does the Vasicek-Basel AIRB model fit the data? Evidence from a long time series of corporate credit rating data, FDIC Working Paper Series, available at: https:// www.fdic.gov/bank/analytical/cfr.

[19] D. Lando and M.S. Nielsen, Correlation in corporate defaults: Contagion or conditional independence? Journal of Financial Intermediation 19(3) (2010), 355-372.

[20] D.X. Li, On default correlation: A copula function approach, Journal of Fixed Income 9(4) (2000), 43-54. doi:10.3905/ jfi.2000.319253.

[21] E. Lütkebohmert, Concentration Risk in Credit Portfolios, Springer, 2009.

[22] R. Merton, On the pricing of corporate debts: The risk structure of interest rates, J. Finance 29 (1974), 449-470.

[23] J. Molins and E. Vives, Long range Ising model for credit risk modeling in homogeneous portfolios, in: AIP Conference Proceedings, Granada, Spain, 2005, pp. 156-161.

[24] Moody's Investors Service, Corporate default and recovery rates, 1920-2010. Moody's Global Credit Policy Special Comment, Annual update.

[25] N. Podlich and M. Wedow, Credit contagion between financial systems, Midwest Finance Association 2012 Annual Meetings Paper.

[26] Y. Roudi, E. Aurell and J. Hertz, Statistical physics of pairwise probability models, Frontiers in Computational Neuroscience 3 (2009), 22.

[27] F. Salmon, Recipe for disaster: The formula that killed Wall Street, Wired Magazine 17(3) (2009).

[28] P. Schönbucher, Information-driven default contagion, ETH Zurich.

[29] M. Steinbacher, M. Steinbacher and M. Steinbacher, Credit contagion in financial markets: A network-based approach, available at: http://mpra.ub.uni-muenchen.de/49616/.

[30] N. Taleb, Antifragile: Things That Gain from Disorder, Random House and Penguin, New York, 2012.

[31] N. Taleb, The Black Swan: The Impact of the Highly Improbable, Random House and Penguin, New York, 2007.

[32] O. Vasicek, Probability of loss on a loan portfolio, Working paper, KMV. Published as: O. Vasicek, Loan portfolio value, Risk (2003), 160-162.

[33] T.C. Wilson, Portfolio credit risk (I), Risk 10(9) (1997), 111117. 